\title{
RITZ METHOD FOR LARGE DEFLECTION OF ORTHOTROPIC THIN PLATES WITH MIXED BOUNDARY CONDITIONS
}

\author{
Madyan A. Al-Shugaa, Husain J. Al-Gahtani, Abubakr E.S. Musa \\ King Fahd University of Petroleum \& Minerals \\ Dhahran 31261, Saudi Arabia \\ madyan.alshugaa@gmail.com,hqahtani@kfupm.edu.sa,bakri083@gmail.com
}

Received: 25 November 2019; Accepted: 4 June 2020

\begin{abstract}
In this paper, the Ritz method is developed for the analysis of thin rectangular orthotropic plates undergoing large deflection. The trial functions approximating the plate lateral and in-plane displacements are represented by simple polynomials. The nonlinear algebraic equations resulting from the application of the concept of minimum potential energy of the orthotropic plate are cast in a matrix form. The developed matrix form equations are then implemented in a Mathematica code that allows for the automation of the solution for an arbitrary number of the trial polynomials. The developed code is tested through several numerical examples involving rectangular plates with different aspect ratios and boundary conditions. The results of all examples demonstrate the efficiency and accuracy of the proposed method.
\end{abstract}

MSC 2010: $74 K 20,82-08,65 N 30$

Keywords: Ritz method, energy method, large-deflection, orthotropic plate bending, mixed boundary conditions, free edges

\section{Introduction}

The application of orthotropic plates has a remarkable contribution in the construction industry such as corrugated plates and laminated composite plates. In many applications, especially if some of the plate edges are free, plates are likely to undergo large deflection. Analytical solutions for the large deflection of orthotropic plates are available for only very few cases involving simply supported and clamped boundary conditions [3-11]. Energy methods offer a more powerful tool for obtaining approximate but fairly accurate analytical solutions to plates with mixed boundary conditions. The Ritz method, in particular, has the advantage of being flexible due to its ability to accommodate trial functions satisfying only the geometric boundary conditions. The application of energy methods to plate analysis can be found in standard textbooks on plates, such as $[1,2,12]$. 
Numerical methods such as the finite difference method [13-16], the finite element method (FEM) [17], the finite strip method [18], the dynamic relaxation method [19], the radial point interpolation method [20] and the method of differential quadrature $[21,22]$ are also possible candidate methods for the current problem. However, all of these numerical methods yield discrete solutions as compared to energy methods which derive the solutions in a functional form. Having the solution in a functional form is of a great advantage, especially for design and optimization purposes.

In this paper, the Ritz method is used to cast the nonlinear equations resulting from the application of the concept of minimum potential energy of the orthotropic plate in a matrix form. The developed matrix form equations are then implemented in a Mathematica code that allows for the automation of the solution for an arbitrary number of trial functions. The developed code is tested through several numerical examples and the accuracy of the obtained solution is verified by comparison with available solutions in the literature and the FEM solutions.

\section{Ritz method formulation for large deflection of orthotropic plates}

The Ritz method is essentially based on minimizing the potential energy in a deformed body [23]. The potential energy of a plate subjected to a load $q$ is defined as the difference between the strain energy stored in the plate during bending and the work done by the applied load. In the following, the strain and potential energies corresponding to large deflection of the orthotropic plate are derived first. Then, the equations resulting from the minimization of the potential energy are cast in a matrix form in order to automate the method and make it easily coded for an arbitrary number of Ritz trial functions.

\subsection{Strain and potential energy}

Consider a thin elastic orthotropic plate subjected to a lateral load $q(x, y)$ causing displacements $u, v$, and $w$ in the $x, y$ and $z$ directions, respectively. The strain energy stored in the plate during bending with consideration of the large deflection strains has mainly two components; the membrane strain energy and the bending strain energy. The membrane strain energy of the plate is defined by

$$
U^{m}=\frac{1}{2} \iint_{A}\left(N_{x} \varepsilon_{x 0}+N_{y} \varepsilon_{y 0}+N_{x y} \gamma_{x y 0}\right) d A
$$

where $\left\{\epsilon_{x 0}, \epsilon_{y 0}, \gamma_{x y 0}\right\}$ are the membrane strains of the middle surface of the plate which are given by 


$$
\left\{\begin{array}{c}
\epsilon_{x 0} \\
\epsilon_{y 0} \\
\gamma_{x y 0}
\end{array}\right\}=\left\{\begin{array}{c}
u_{, x}+\frac{1}{2}\left(w_{, x}\right)^{2} \\
v_{, y}+\frac{1}{2}\left(w_{, y}\right)^{2} \\
u_{, y}+v_{, x}+w, x w, y
\end{array}\right\}
$$

and $\left\{N_{x}, N_{y}, N_{x y}\right\}$ are the membrane forces which are related to strains by

$$
\left\{\begin{array}{c}
N_{x} \\
N_{y} \\
N_{x y}
\end{array}\right\}=\left[\begin{array}{ccc}
A_{11} & A_{12} & 0 \\
A_{12} & A_{22} & 0 \\
0 & 0 & A_{66}
\end{array}\right]\left\{\begin{array}{c}
\epsilon_{x 0} \\
\epsilon_{y 0} \\
\gamma_{x y 0}
\end{array}\right\}
$$

where $[A]=\int_{-t / 2}^{t / 2}[Q] d z$ is the extensional stiffness matrix and matrix $\mathrm{Q}$ is given by

$$
\left[\begin{array}{ccc}
Q_{11} & Q_{12} & 0 \\
Q_{12} & Q_{22} & 0 \\
0 & 0 & Q_{66}
\end{array}\right]=\frac{1}{1-v_{x y} v_{y x}}\left[\begin{array}{ccc}
E_{x} & v_{y x} E_{x} & 0 \\
v_{x y} E_{y} & E_{y} & 0 \\
0 & 0 & G_{x y}\left(1-v_{x y} v_{y x}\right)
\end{array}\right]
$$

Substitution of Equation (2) along with Equation (3) in Equation (1) yields the membrane strain energy of orthotropic plate in terms of displacements derivatives, i.e.:

$$
\begin{aligned}
U^{m}=\frac{1}{8} \iint_{A}\left(A_{11}\right. & \left(2 u_{\prime x}+w_{\prime x}^{2}\right)^{2}+A_{22}\left(2 v_{1 y}+w_{\prime y}^{2}\right)^{2} \\
& +2 A_{12}\left(2 u_{\prime x}+w_{\prime x}^{2}\right)\left(2 v_{\prime y}+w_{\prime y}^{2}\right) \\
& \left.+4 A_{66}\left(u_{1 y}+v_{1 x}+w_{\prime x} w_{\prime y}\right)^{2}\right) d A
\end{aligned}
$$

The bending strain energy is defined by

$$
U^{b}=\frac{1}{2} \iint_{A}\left(M_{x} \kappa_{x}+M_{y} \kappa_{y}+M_{x y} \kappa_{x y}\right) d A
$$

where $\left\{\kappa_{x}, \kappa_{y}, \kappa_{x y}\right\}$ are the components of the plate curvatures which are given by

$$
\left\{\begin{array}{c}
\kappa_{x} \\
\kappa_{y} \\
\kappa_{x y}
\end{array}\right\}=\left\{\begin{array}{l}
w, x x \\
w, y y \\
w, x y
\end{array}\right\}
$$

and $\left\{M_{x}, M_{y}, M_{x y}\right\}$ are the bending moments which are related to curvatures by

$$
\left\{\begin{array}{c}
M_{x} \\
M_{y} \\
M_{x y}
\end{array}\right\}=\left[\begin{array}{ccc}
D_{11} & D_{12} & 0 \\
D_{12} & D_{22} & 0 \\
0 & 0 & D_{66}
\end{array}\right]\left\{\begin{array}{c}
\kappa_{x} \\
\kappa_{y} \\
2 \kappa_{x y}
\end{array}\right\}
$$

where $[D]=\int_{-t / 2}^{t / 2}[Q] z d z$ is the bending stiffness matrix. 
Substitution of Equation (6) along with Equation (7) in Equation (5) yields the bending strain energy of orthotropic plate in terms of displacements, i.e.:

$$
U^{b}=\frac{1}{2} \iint_{A}\left(D_{11} w_{\prime x x}^{2}+D_{22} w_{1 y y}^{2}+2 D_{12} w_{1 x x} w_{1 y y}+4 D_{66} w_{1 x y}^{2}\right) d A
$$

The work done by the external force can also be expressed

$$
\mathrm{W}=\iint_{A} q w d A
$$

Finally, the potential energy is given by

$$
\Pi=U^{m}+U^{b}-W
$$

\subsection{Ritz method in matrix form}

For convenience, the derivation of the equations will be performed using indicial notation. The first step in formulating the Ritz method is to approximate the solutions for $u, v$, and $w$ by the following

$$
\begin{array}{ll}
u(x, y)=C_{i}^{u} \phi_{i}(x, y), & i=1, N_{u} \\
v(x, y)=C_{j}^{v} \chi_{j}(x, y), & j=1, N_{v} \\
w(x, y)=C_{k}^{w} \psi_{k}(x, y), & k=1, N_{w}
\end{array}
$$

where the trial functions $\phi_{i}, \chi_{j}$ and $\psi_{k}$ are selected to satisfy only the geometric boundary conditions; $N_{u}, N_{v}$, and $N_{w}$ are the number of terms for each of the three trial functions; and the coefficients $C_{i}^{u}, C_{j}^{v}$ and $C_{k}^{w}$ are to be determined based on the principle of minimum potential energy, i.e.:

$$
\begin{array}{lll}
\Pi, C_{r}^{u}=U_{, C_{r}^{u}}^{m}+U_{, C_{r}^{u}}^{b}-W, C_{r}^{u}=0, & r=1, N_{u} \\
\Pi, C_{s}^{v}=U_{, C_{s}^{v}}^{m}+U_{, C_{s}^{v}}^{b}-W, C_{s}^{v}=0, & s=1, N_{v} \\
\Pi, C_{t}^{w}=U_{, C_{t}^{w}}^{m}+U_{, C_{t}^{w}}^{b}-W, C_{t}^{w}=0, & t=1, N_{w}
\end{array}
$$

Inspecting the dependency of the energy quantities, $U^{m}, U^{b}$ and $\mathrm{W}$, on the constants $C_{i}^{u}, C_{j}^{v}$ and $C_{k}^{w}$ and carrying out the lengthy task of all differentiations in the system of Equation (12) results in the following equations written in matrix form

$$
\left[\begin{array}{ccc}
K_{11}^{r i} & K_{12}^{r j} & 0 \\
K_{21}^{s i} & K_{22}^{s j} & 0 \\
0 & 0 & K_{33}^{t k}
\end{array}\right]\left[\begin{array}{c}
C_{i}^{u} \\
C_{j}^{v} \\
C_{k}^{w}
\end{array}\right]=\left[\begin{array}{c}
0 \\
0 \\
\iint_{A} q \psi_{t} d A
\end{array}\right]+\left[\begin{array}{c}
N L F 1_{r}\left(C_{k}^{w}\right) \\
N L F 2_{s}\left(C_{k}^{w}\right) \\
N L F 3_{t}\left(C_{i}^{u}, C_{j}^{v}, C_{k}^{w}\right)
\end{array}\right]
$$


where:

$$
\begin{gathered}
K_{11}^{r i}=\iint_{A}\left(A_{11} \phi_{i, x} \phi_{r, x}+A_{66} \phi_{i, y} \phi_{r, y}\right) d A \\
K_{12}^{r j}=\iint_{A}\left(A_{12} \chi_{j, y} \phi_{r, x}+A_{66} \chi_{j, x} \phi_{r, y}\right) d A \\
K_{21}^{s i}=\iint_{A}\left(A_{12} \phi_{i, x} \chi_{s, y}+A_{66} \phi_{i, y} \chi_{s, x}\right) d A \\
K_{22}^{s j}=\iint_{A}\left(A_{22} \chi_{j, y} \chi_{s, y}+A_{66} \chi_{j, x} \chi_{s, x}\right) d A \\
K_{33}^{t k}=\iint_{A}\left(\psi_{t, x x}\left(D_{11} \psi_{k, x x}+D_{12} \psi_{k, y y}\right)+\psi_{t, y y}\left(D_{22} \psi_{k, y y}+D_{12} \psi_{k, x x}\right)\right. \\
\left.+4 D_{66} \psi_{t, x y} \psi_{k, x y}\right) d A \\
N L F 1_{r}=-\frac{1}{2} C_{k}^{w} C_{l}^{w} \iint_{A}\left(\phi_{r, x}\left(A_{11} \psi_{k, x} \psi_{l, x}+A_{12} \psi_{k, y} \psi_{l, y}\right)\right. \\
\left.+A_{66} \phi_{r, y} \psi_{k, x} \psi_{l, y}\right) d A \\
\left.+A_{66} \chi_{j, x}\left(\psi_{k, x} \psi_{t, y}+\psi_{k, y} \psi_{t, x}\right)\right) d A \\
+C_{i}^{u} C_{k}^{w} \iint_{A}\left(\phi_{i, x}\left(A_{11} \psi_{k, x} \psi_{t, x}+A_{12} \psi_{k, y} \psi_{t, y}\right)\right. \\
\left.+\left(\psi_{k, x} \psi_{t, y}+\psi_{k, y} \psi_{t, x}\right)\right) d A \\
N L F 3_{t}=-\frac{1}{2} C_{k}^{w} C_{l}^{w} C_{m}^{w} \iint_{A}\left(A_{11} \psi_{k, x} \psi_{l, x} \psi_{m, x} \psi_{t, x}+A_{22} \psi_{k, y} \psi_{l, y} \psi_{m, y} \psi_{t, y}\left(\psi_{k, y} \psi_{l, y} \psi_{m, x} \psi_{t, x}\right.\right. \\
+-\frac{1}{2} C_{k}^{w} C_{l}^{w} \iint_{A}\left(\chi_{s, y}\left(A_{22} \psi_{k, y} \psi_{l, y}+A_{12} \psi_{k, x} \psi_{l, x}\right)\right. \\
\left.+2 A_{66} \chi_{s, x} \psi_{k, x} \psi_{l, y}\right) d A
\end{gathered}
$$

where the indices take values according to the following: $r, i=1, N_{u}, s, j=1, N_{v}$ and $k, l, m, t=1, N_{w}$. 
The system of equations represented by Equation (13) contains a number of $\left(N_{u}+N_{v}+N_{w}\right)$ nonlinear equations in terms of the same number of unknown coefficients. The computations of all integrals and the solution of the resulting nonlinear equations have been carried out using Wolfram Mathematica [24]. The solution of the system of equations has been performed using the built-in function „FindRoot” of Mathematica, which is substantially easier than the well-known iterative procedure.

\section{Numerical examples}

The accuracy and versatility of the proposed method is examined through three numerical examples representing three different set of boundary conditions: (a) all sides are simply supported and restrained against in-plane movement (SSSS), (b) two opposite sides are simply supported and restrained against in-plane movement and the other two are free to move in all directions (SFSF) and (c) three sides are simply supported and restrained against in-plane movement and the fourth edge is free to move both laterally and in the in-plane directions (SSSF).
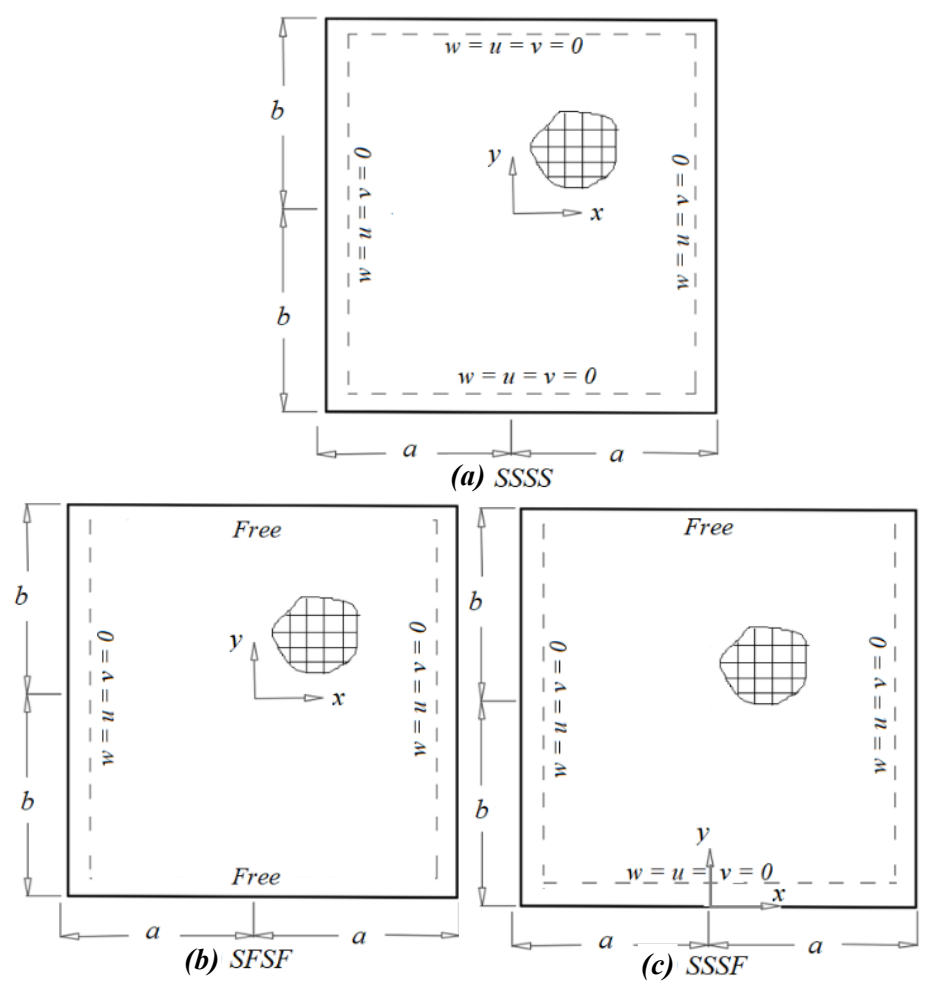

Fig. 1. Rectangular orthotropic plates with sides of $(2 a \times 2 b)$

The geometry and boundary conditions for the above three cases are shown in Figure 1. To utilize symmetry, the origin of coordinates is placed at the center of 
the plate for the first two examples. For the third example, the origin is moved to the center of the bottom edge. The dimensions of the plate are $(2 a \times 2 b)$ and the analysis is carried out for three aspect ratios: $b / a=1 / 2,2 / 3 \& 1$. To certify the adequacy of the proposed method, the first case (SSSS) has been solved for square orthotropic plate and compared with the obtained results by Reddy [17]. The results were obtained graphically similar to Reddy's presentation for fair comparison. Then, the nondimensional graphical results of the deflection and stresses for different aspect ratios were obtained and verified against the FEM. For the other two cases involving free edges (SFSF, SSSF), there are no previously published solutions and therefore, their results are verified against FEM solutions only. The same plate properties assumed by Reddy [17] have been used here for all the examples. These are: $a=6 \mathrm{in}, t=0.138 \mathrm{in}, E_{x}=3 \times 10^{6} \mathrm{psi}, E_{y}=1.28 \times 10^{6} \mathrm{psi}$, $G_{x y}=0.37 \times 10^{6} p s i$ and $v_{x y}=0.32$.

The FEM analysis was performed using ABAQUS software with an extremely fine mesh having a maximum element size of a/60. The element type is STRI3 which considers geometrical nonlinearity based on classical plate theory [25].

\section{Example 1. Uniformly loaded SSSS rectangular orthotropic plates}

As per Ritz method requirements, the trial functions are required to satisfy the essential boundary conditions. Thus, the solution can be obtained by employing the following trial polynomials for this case of boundary conditions:

$$
\begin{aligned}
& u=\sum_{j=0}^{m} \sum_{i=0}^{n} C_{i j}^{u}\left(a^{2}-x^{2}\right)\left(b^{2}-y^{2}\right) x^{2 i+1} y^{2 j} \\
& v=\sum_{j=0}^{m} \sum_{i=0}^{n} C_{i j}^{v}\left(a^{2}-x^{2}\right)\left(b^{2}-y^{2}\right) x^{2 i} y^{2 j+1} \\
& w=\sum_{j=0}^{m} \sum_{i=0}^{n} C_{i j}^{w}\left(a^{2}-x^{2}\right)\left(b^{2}-y^{2}\right) x^{2 i} y^{2 j}
\end{aligned}
$$

The criterion for truncating the number of polynomial terms was based on convergence to the fine mesh-FEM solution. The convergence study showed that the complete agreement with FEM solution can be obtained with $(m=n=3)$ results in 16, 16 and 16 unknown coefficients for $u, v$, and $w$, respectively. This problem has been solved by Reddy [17] for a square plate $(a=b=6 \mathrm{in})$. The convergence analysis in Table 1 shows that the deflection converges rapidly with few terms while the normal and bending stresses required more term to get converge $(m=n=3)$. The comparisons of the present solution with those obtained by Reddy [17] and FEM are shown in Figures $2 \mathrm{a} \& 2 \mathrm{~b}$ for central deflection and maximum stresses, respectively. The Figures show perfect agreement among the three solutions. 
Table 1. Convergence analysis results at center point for square SSSS plate, $q=2.6$

\begin{tabular}{|c|c|c|c|c|c|}
\hline $\mathrm{m}, \mathrm{n}$ & $w / t$ & $\sigma m_{x} \frac{\mu(2 b)^{2}}{E_{y} t^{2}}$ & $\sigma m_{y} \frac{\mu(2 b)^{2}}{E_{y} t^{2}}$ & $\sigma b_{x} \frac{\mu(2 b)^{2}}{E_{y} t^{2}}$ & $\sigma b_{y} \frac{\mu(2 b)^{2}}{E_{y} t^{2}}$ \\
\hline 1 & 0.0925 & 0.0300 & 0.0136 & 0.7471 & 0.3702 \\
\hline 2 & 1.6094 & 8.7776 & 4.1640 & 14.7027 & 6.7756 \\
\hline 3 & 1.6021 & 8.8005 & 4.2804 & 13.4794 & 5.5947 \\
\hline 4 & 1.6021 & 8.8082 & 4.2870 & 13.4536 & 5.5838 \\
\hline 5 & 1.6021 & 8.8085 & 4.2868 & 13.4414 & 5.5809 \\
\hline
\end{tabular}
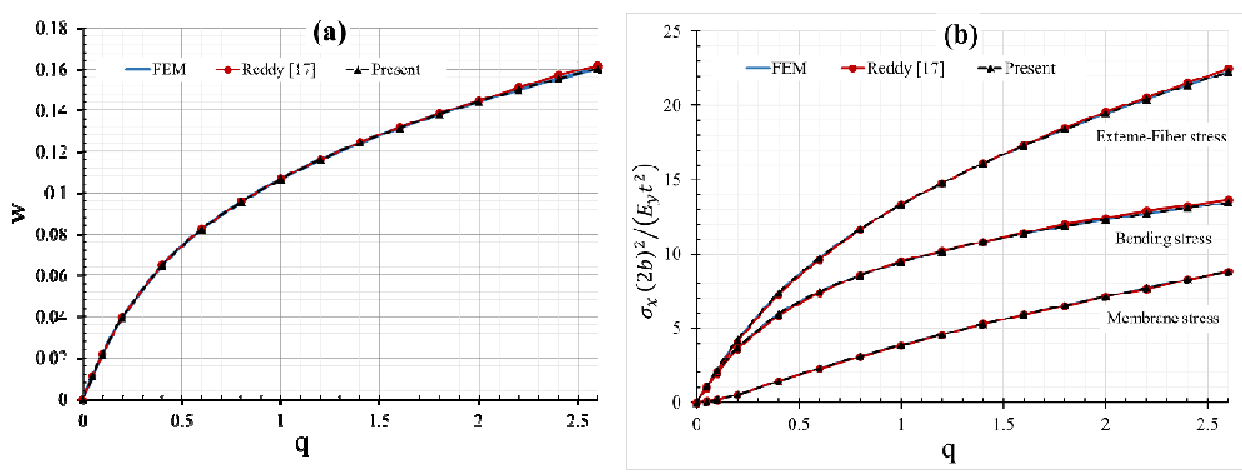

Fig. 2. Uniformly loaded SSSS-orthotropic square plates: a) central deflections, b) membrane and exterme stress at $(x=0, y=0)$

For the plate with aspect ratios $\alpha=1 / 2 \& 2 / 3$, no published solutions are available and, therefore, the present solution is compared with the FEM only. The normalized central plate deflection (w/t) and stress $\left(\mu \sigma_{x}(2 b)^{2} /\left(E_{y} t^{2}\right)\right)$, where $\mu=\left(1-v_{x y} v_{y x}\right)$, are plotted versus the normalized load $\left(q(2 b)^{4} /\left(E_{y} t^{4}\right)\right)$ in Figures $3 \mathrm{a}$ and $3 \mathrm{~b}$ for the deflection and stresses, respectively. The Figures confirm the perfect agreement between the present solutions and those obtained by the FEM.
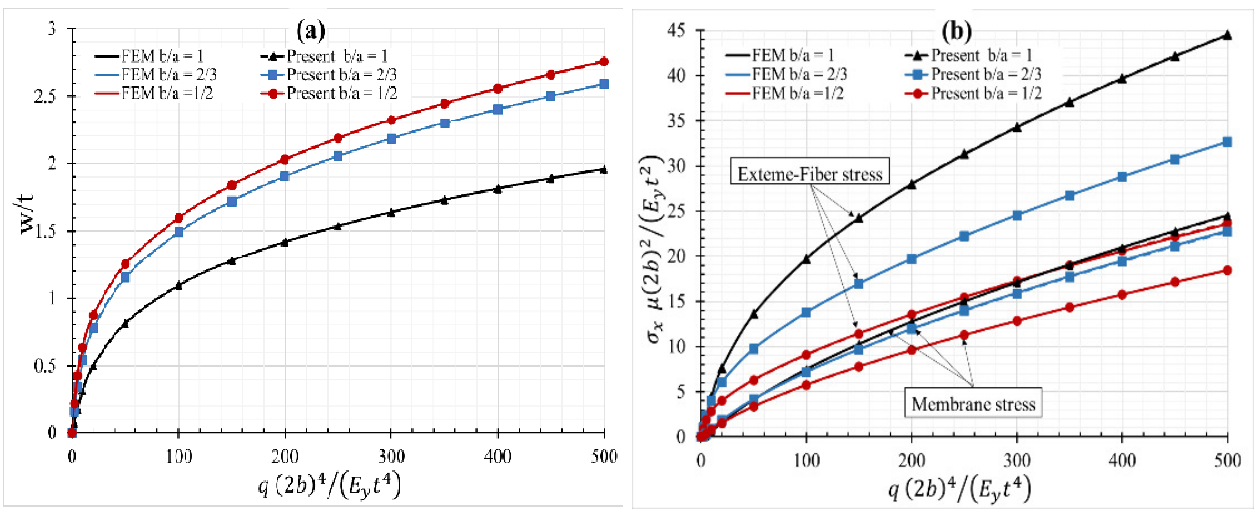

Fig. 3. Uniformly loaded SSSS-orthotropic plates: a) central deflections, b) membrane and exterme stress at $(\mathrm{x}=0, \mathrm{y}=0)$ 


\section{Example 2. Uniformly loaded SFSF rectangular orthotropic plate}

The plate is simply supported, prevented from in-plane movement at the edges $( \pm a, y)$ and free to move at the edges $(x, \pm b)$. The trial functions become:

$$
\begin{aligned}
& u=\sum_{j=0}^{2} \sum_{i=0}^{2} C_{i j}^{u}\left(a^{2}-x^{2}\right) x^{2 i+1} y^{2 j} \\
& v=\sum_{j=0}^{2} \sum_{i=0}^{2} C_{i j}^{v}\left(a^{2}-x^{2}\right) x^{2 i} y^{2 j+1} \\
& w=\sum_{j=0}^{3} \sum_{i=0}^{4} C_{i j}^{w}\left(a^{2}-x^{2}\right) x^{2 i} y^{2 j}
\end{aligned}
$$

The verification of the proposed method is performed against the FEM solution for different aspect ratios: $\alpha=b / a=1 / 2,2 / 3,1$. Figures 4 and 5 show the results for deflection and stresses at the critical points of plate, namely: the center and the middle of the free edges. The results presented in all Figures show a perfect agreement between the two solutions.
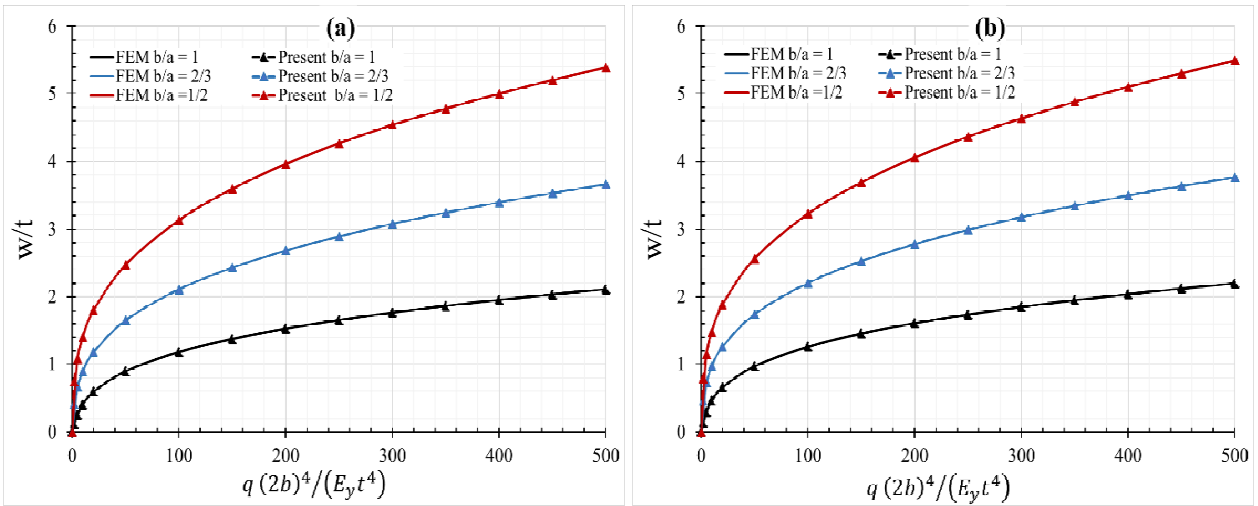

Fig. 4. Deflection for uniformly loaded SFSF-orthotropic plate: a) at center $(0,0)$, b) at mid-free edges $(0, \pm b)$
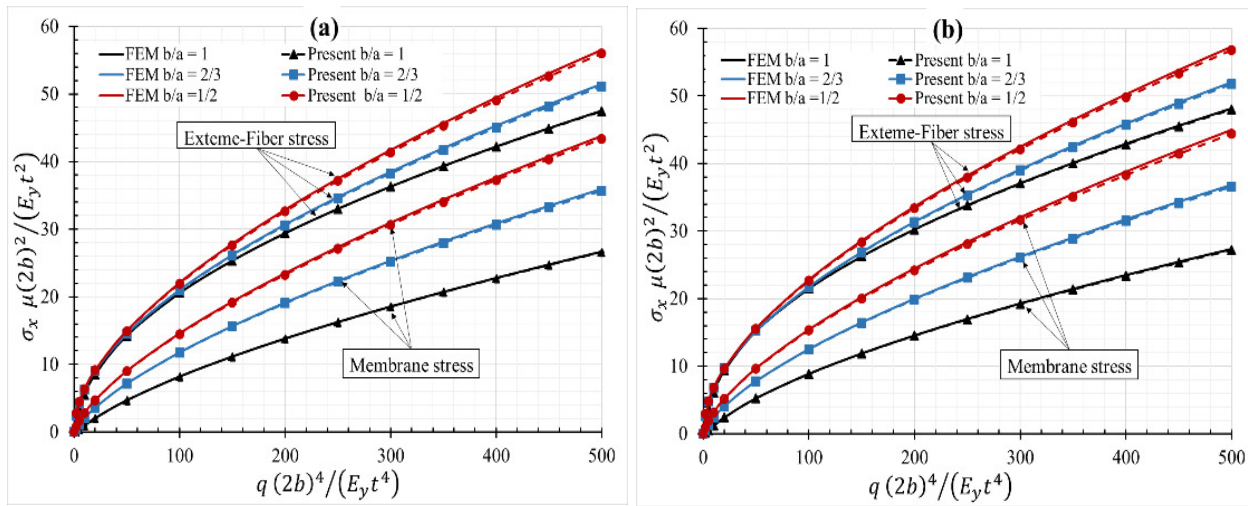

Fig. 5. Membrane and extreme-fiber stress for uniformly loaded SFSF- orthotropic plate: a) at center $(0,0), b)$ at free edges $(0, \pm b)$ 


\section{Example 3. Uniformly loaded SSSF rectangular orthotropic plate}

In this example, the rectangular plate is simply supported and prevented from in-plane movements while the fourth edge at $(x, 2 b)$ is free to move in all directions. The origin of this plate is at the middle of the bottom (simply supported) edge. The approximate expressions for $u, v$ and $w$ are given by:

$$
\begin{aligned}
& u=\sum_{j=0}^{2} \sum_{i=0}^{4} C_{i j}^{u}\left(a^{2}-x^{2}\right) x^{2 i+1} y^{j+1} \\
& v=\sum_{j=0}^{2} \sum_{i=0}^{4} C_{i j}^{v}\left(a^{2}-x^{2}\right) x^{2 i} y^{j+1} \\
& w=\sum_{j=0}^{2} \sum_{i=0}^{6} C_{i j}^{v}\left(a^{2}-x^{2}\right) x^{2 i} y^{j+1}
\end{aligned}
$$

Figure 6 shows the results for the lateral deflection evaluated at the center and at the middle of the free edge, while Figure 7 shows the corresponding membrane and extreme-fiber stresses. The excellent agreements between all curves for the two solutions confirm the accuracy of the proposed formulation for the Ritz method.
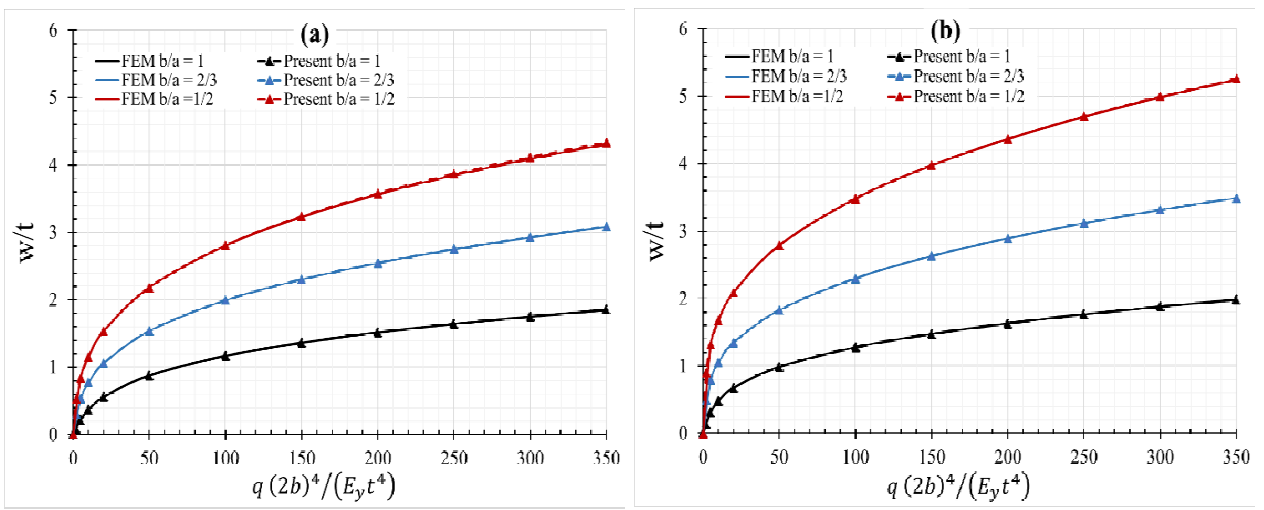

Fig. 6. Deflection for uniformly loaded SSSF-orthotropic plate: a) at center $(0, b)$, b) at free edges $(0,2 b)$
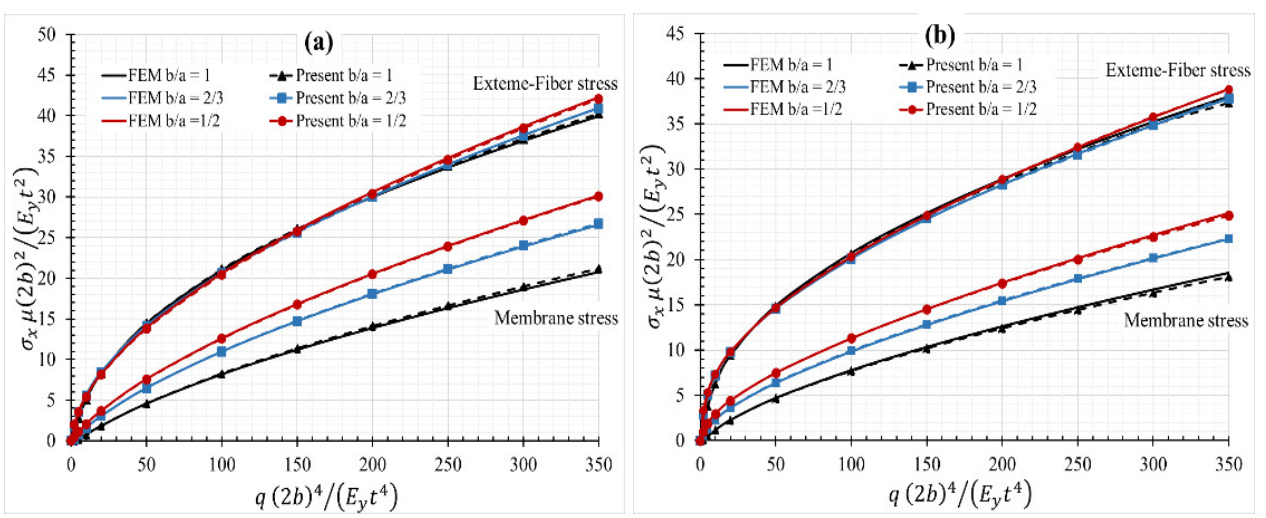

Fig. 7. Membrane and extreme-fiber stress for uniformly loaded SSSF-orthotropic plate: a) at center $(0, b), b)$ at free edges $(0,2 b)$ 
For the particular case of $\mathrm{b} / \mathrm{a}=1$, a numerical comparison between the results of the present solutions and FEM solutions for the above three examples is given in Table 2 which gives the normalized central deflection and stress for different loading values. Again, the numerical comparison confirms the accuracy of the present method.

Table 2. Normalized central deflection and stress versus normalized load for $\mathrm{d} b / \mathrm{a}=1$

\begin{tabular}{|c|c|c|c|c|c|c|c|}
\hline \multirow{2}{*}{$q \frac{(2 b)^{4}}{E_{y} t^{4}}$} & \multirow{2}{*}{ Expression } & \multicolumn{2}{|c|}{ SSSS } & \multicolumn{2}{|c|}{ SFSF } & \multicolumn{2}{|c|}{ SSSF } \\
\hline & & FEM & Ritz & FEM & Ritz & FEM & Ritz \\
\hline 100 & \multirow{3}{*}{$\mathrm{w} / \mathrm{t}$} & 1.0949 & 1.0947 & 1.1824 & 1.1823 & 1.1709 & 1.17132 \\
\hline 200 & & 1.4231 & 1.4227 & 1.5291 & 1.5289 & 1.5193 & 1.52025 \\
\hline 300 & & 1.6433 & 1.6434 & 1.7672 & 1.7670 & 1.7552 & 1.75627 \\
\hline 100 & \multirow{3}{*}{$\sigma_{x} \frac{\mu(2 b)^{2}}{E_{y} t^{2}}$} & 19.7120 & 19.7135 & 20.5499 & 20.5535 & 20.9871 & 21.0876 \\
\hline 200 & & 27.9968 & 27.9982 & 29.4061 & 29.3962 & 29.9903 & 30.1909 \\
\hline 300 & & 34.3009 & 34.3228 & 36.3072 & 36.2757 & 36.8951 & 37.1475 \\
\hline
\end{tabular}

\section{Conclusions}

The Ritz method is presented for the solution of large deflection of thin rectangular orthotropic plates governed by von Karman equations. The nonlinear equations resulting from the application of the concept of minimum potential energy of the orthotropic plate are first cast in a matrix form which facilitates the implementation of Ritz method in a simple Mathematica code capable of accommodating as many polynomial terms as required to achieve convergence of the solution. The derived matrix form is capable of handling different boundary conditions including free edges and different degrees of orthotropy. The results of the numerical examples confirm the advantage of automating the Ritz method in terms of its accuracy and capability in handling the present complicated problem.

\section{Acknowledgement}

The authors gratefully acknowledge the support provided by King Fahd University of Petroleum \& Minerals (KFUPM) for this work.

\section{References}

[1] Leknitskii, S. (1968). Anisotropic Plates (2nd edn). New York: Gordon and Breach.

[2] Whitney, J.M. (1987). Structural Analysis of Laminated Anisotropic Plates. Taylor \& Francis Group, LLC.

[3] Chia, C.Y. (1972). Finite deflections of uniformly loaded, clamped, rectangular, anisotropic plates. AIAA Journal, 10(11), 1399-1400. DOI: 10.2514/3.50383. 
[4] Banerjee, B., \& Datta, S. (1981). A new approach to an analysis of large deflections of thin elastic plates. Int. J. Non-Linear. Mech., 16(1), 47-52.

[5] Gorji, M. (1986). On large deflection of symmetric composite plates under static loading. Proc. Lnstn. Mech. Engrs., 200(C1), 13-19.

[6] Prabhakara, M.K., \& Chia, C.Y. (1973). Large deflections of rectangular orthotropic plates under combined transverse and in-plane loads. J. Mech. Eng. Sci., 15(5), 346-350.

[7] Chiat, C.Y., \& Prabhakara, M.K. (1975). Nonlinear analysis of orthotropic plates. J. Mech. Eng. Sci., 17(3), 133-138.

[8] Little, G.H. (1987). Efficient large deflection analysis of rectangular orthotropic plates by direct energy minimisation. Comp. \& Struct., 26(5), 871-884.

[9] Little, G. H. (1988). Large deflection analysis of orthotropic plates adaptation of coan's method. Int. J. Mech. Sci., 30(I), 31-42.

[10] Gordon, H.L. (1990). Large deflections of orthotropic plates under pressure. J. Eng. Mech., ASCE, 115(12), 2601-2620.

[11] Yeh, F.H., \& Liu, W.H. (1991). Nonlinear analysis of rectangular orthotropic plates. Int. J. Mech. Sci., 33(7), 563-578.

[12] Timoshenko, S.P., \& Woinowsky-Krieger, S. (1959). Theory of Plates and Shells. McGraw-Hill.

[13] Basu, A.K., \& Chapman, J.C. (1966). Large deflexion behaviour of transversely loaded rectangular orthotropic plates. Roc. Instn. Civ. Engrs., 35(6927), 233-234.

[14] Basu, A.K., \& Chapman, J.C. (1967). Large deflection behaviour of transversely loaded rectangular orthotropic plates. Roc. Instn. Civ. Engrs., (6927), 79-110.

[15] Aalami, B., \& Chapman, J. (1969). Large deflexion behaviour of rectangular orthotropic plates under transverse and in-planeloads. ICE Proceedings, 42(July), 347-382.

[16] Yeh, Y., Chi, C., \& Jang, M. (2007). Using finite difference and differential transformation method to analyze of large deflections of orthotropic rectangular plate problem. Appl. Math. Comput., 190, 1146-1156.

[17] Reddy, J.N. (2003). Mechanics of Laminated Composite Plates and Shells: Theory and Analysis (2nd ed.). CRC Press.

[18] Abayakoon, S.B. (1987). Large deflection elastic-plastic analysis of plate structures by the finite strip method, PhD thesis. The University of British Columbia.

[19] Kadkhodayan, M., Erfani Moghadam, A., Turvey, G.J., \& Alamatian, J. (2012). A DXDR large deflection analysis of uniformly loaded square, circular and elliptical orthotropic plates using non-uniform rectangular finite-differences. J. Mech. Sci. Technol., 26(10), 3231-3242.

[20] Liu, X.Z.G.R., Zhong, K.Y.D.Z.H., \& Han, G.Y.L.X. (2008). Geometric nonlinear analysis of plates and cylindrical shells via a linearly conforming radial point interpolation method. Comput. Mech., 48, 133-144.

[21] Bert, C.W., Jang, S.K., \& Striz, A.G. (1989). Nonlinear bending analysis of orthotropic rectangular plates by the method of differential quadrature. Comput. Mech., 5, 217-226.

[22] Chen, W., Shu, C., He, W., \& Zhong, T. (2000). The application of special matrix product to differential quadrature solution of geometrically nonlinear bending of orthotropic rectangular plates. Comp. Struct. 74, 65-76.

[23] Langhaar, L.H. (1962). Energy Methods in Applied Mechanics. New York: John Wiley \& Sons, Inc.

[24] Wolfram Research. (2018). Mathematica. Version 11. Champaign, Illinois: Wolfram Research, Inc.

[25] Simulia, D.S. (2013). ABAQUS 6.13 Analysis User's Guide. Online Documentation. 